\title{
Urology after COVID-19: the post-apocalypse
}

\begin{abstract}
COVID-19 pandemic has overwhelmed health care systems worldwide. Urologists are part of the system that needs to recover. However, we should be aware that we cannot simply return to work as usual. The after effect of this crisis will last for a period of time after all is declared over. We need to learn our lessons and to start proactively planning how to resume back our elective service provision in order to survive the post-COVID-19 era and be ready for any similar events in the future.
\end{abstract}

Keywords: COVID-19, pandemic, urology, procedures, theatres
Volume 8 Issue 4 - 2020

\author{
Elsayed Desouky',2 \\ 'Urology Department, Wexham Park NHS Hospital, UK \\ ${ }^{2}$ Urology Department, Alexandria Main University Hospital, \\ Egypt
}

Correspondence: Elsayed Desouky, Wexham Park Hospital, Berkshire, UK, Email e.desouky@nhs.net

Received: July 24, 2020 | Published: August 31, 2020
Abbreviations: WHO, world health organization; ICU, intensive care unit; PPE, personal protective equipment; EAU, european association of urology; AUA, american association of urology

\section{Introduction}

As the coronavirus takes over the globe, countries have locked down, transforming into ghost cities. No pandemic or plague or natural disaster has killed off humanity's ability to survive. Not the Black Plagues of the $14^{\text {th }}$ century or London's cholera epidemic in the 1850 s, or even 1918's Spanish Flu. That's because humans' survival instinct is just too strong. We will get through this pandemic, too. Hospitals will resume back the usual activities; outpatient clinics, wards and theatres etc. all will come back. However, when we do, hospital managers and health care professionals should be aware that we cannot simply return to work as usual. This will take time to recover from the crisis and to initiate a safe route to recover our elective activities in the post COVID-19 era. After, we will need plans to prepare for future pandemics. Below are some highlights on tracking of the current pandemic, presenting some key measures to prepare our practice for the next phase of the coronavirus crisis and beyond.

\section{Patients}

Several studies have been published regarding the clinical picture of COVID-19 and the immune response to SARS-CoV-2. However, the time sequence of clinical presentation, virus shedding kinetics and contagiousness which are essential input for infection control strategy planning, have been less discussed. ${ }^{1}$ Prolonged virus shedding even after seroconversion in patients with COVID-19 has been reported. ${ }^{2}$ Viral load has been detected in respiratory droplets, feces and serum. To date, it is uncommon to detect the viral load in urine samples. ${ }^{3,4}$ How long the patient should be self-isolating after recovery is not well defined yet. In addition, the risk of re-infection/re-activation is still a concern. ${ }^{5}$ The World Health Organization (WHO) reported that there is no evidence that people who have recovered from coronavirus and have antibodies are protected against a second infection. WHO warned against issuing "immunity passports" or "risk free certificates" that would allow people to work or travel, claiming that relying on antibodies at this stage of the pandemic could increase the spread of the virus as countries look to reopen after quarantines and other mitigation efforts. ${ }^{6}$ All these issues need to be taken into consideration upon planning resumption of clinical activity. Some outpatient procedures e.g. flexible cystoscopy, catheter change or intra-vesical BCG instillation, can be resumed gradually considering the following;

I. Physical attendance into hospital premises, still need to be minimized as much as possible in the initial phase

II. Screening patients via questioning through a telephone clinic about symptoms of COVID-19 before attendance is essential to act accordingly

III. Allowing plenty of time intervals between patients to follow social distancing rule

IV. Asking patients to attend on their own if at all possible

V. Patients and clinicians need to follow the appropriate infection control measures

VI. Screening swabs for patients before inpatient hospital admission should be considered

VII. Possible extended / additional work afterhours or on weekends in order to cope with the backlog

VIII. Possible referral of semi-urgent cases to other hospitals, sometimes at a distance, with less COVID-19 caseload or less restriction.

\section{Urologists}

Physical and mental stresses, to which health care professionals are currently exposed, are enormous. The rapid surge of the infection forced all hospitals to shift quickly to dedicated "COVID structures." Almost all elective procedures have been postponed and only emergency procedures continued. Regular hospital wards are now called COVID wards. Every single medical professional is involved. Surgeons have gone back to school to study the respiratory system, urologists are now applying noninvasive mechanical ventilation, and cardiologists have become intensivists. The main target is as in our Oath: Heal if you can, treat as much as possible, and always assist. ${ }^{7}$ Falling sick, having to self-isolate at times and worrying about contracting infection to your beloved ones at home, let alone the grief reaction to a colleague who passed away, all these are not easy issues to come over and definitely need time to recover.

With the tsunami of COVID-19, self-isolation can be a special opportunity to complete research that one could never find time among the busy schedule time for clinical work; for a trainee, it may be time to study the textbooks that has been not opened. ${ }^{8}$ COVID-19 presents 
a 'Novel' virus with 'Old' lessons. This crisis represents a call for action to proactively start effective planning to improve mass casualty management preparedness in general, and for COVID-19 in particular. Global Health cannot continue to consider mass medical emergencies as extraordinary situations when they are part of the natural course of things. As natural parts of the common life, Global Health must be prepared to face them at the best of our possibilities. ${ }^{9,10} \mathrm{We}$ - urologists are part of this call. This is a timely reminder to urologists of our responsibility as doctors first. COVID-19, emergency medicine and preventive medicine may have been removed far from our training schedule, but we need always to remember our Hippocratic Oath that we will always support those who seek our help. We have chosen our specialty in genitourinary surgery but still, in times of need, urologists have a role to play in public health and serving the society. ${ }^{11}$

\section{Theatres}

No one would expect theatre elective surgical lists to resume back to normal immediately after the pandemic is over. There will be time needed to disinfect and to deep clean all rooms, equipment and ventilators as well as to reorganize the remaining logistics. We should also take into consideration our colleagues who have been involved in the frontline, being deployed from theatres elsewhere for alternative roles; anesthetists into intensivists, recovery nurses into intensive care units (ICU) and even scrub nurses into wards. When the first wave of this pandemic is behind us, the pent-up patient demand for surgical and procedural care may be massive and health care professionals must be prepared to meet this demand. Many patients have had their surgeries postponed due to the pandemic. Physician and facility readiness to resume elective surgery will vary by location. The following are the main points to consider when planning for service resumption; ${ }^{12}$

I. There should be a significant stable reduction in the rate of new COVID-19 cases in the relevant geographic area for at least 14 days

II. Facilities shall have appropriate number of ICU and non-ICU beds, personal protective equipment (PPE), ventilators and trained staff

III. Facilities should establish a prioritization strategy appropriate to the immediate patient needs

IV. Facilities should adopt policies addressing care issues specific to COVID-19 and the postponement of surgical scheduling

V. Facilities should have and implement a social distancing policy for staff, patients and patient visitors.

As resumption of theatre activities will take time to fully recover, the need to triage our patients in a pragmatic approach will continue for a while even after all of this is over. The simplest way to do that for resumption of activity is to mirror image your own triaging schedule ${ }^{13}$ you have been following during this pandemic time. This will give priority to emergency cases and urgent cancer patients to be sorted first (Table 1). To maintain patient's safety, we will have to consider the after effect of the pandemic on all perioperative circumstances which may affect the patient's care. This needs to be clearly and truthfully explained to the patient beforehand during the consultation in order to obtain an 'informed consent'. ${ }^{13}$

\section{Telemedicine}

The current coronavirus (COVID-19) pandemic is reminding us of the importance of using telehealth to deliver care, especially as means of reducing the risk of cross-infection caused by close contact. Telehealth first needs to become a routinely used part of our health system in order to be effective as part of an emergency response. Hence, it is time to step back and ask why telehealth is not mainstreamed. ${ }^{14}$ In a recent publication about 'Tele-urology' ${ }^{15}$ it was possible to keep a relatively high level (55\%) of direct consultation via telephone. A vast majority of face-to-face visits regarded sensitive (suspected recurrent or new onset malignancy, $46 \%$ ) or potentially dangerous clinical conditions (severe urinary symptoms or complicated urinary stones or infection, $28 \%$ ). The rate of face-to-face visit decreased from $63 \%$ to $9 \%$ during weeks 1 and 4 after the lockdown, respectively.

Continuous medical education as well has been disrupted by the pandemic but it has been saved, at least partially, being part of the scope of telemedicine. The 2020 annual meeting of EAU, AUA as well as other national and international urological societies has been cancelled. These will be replaced by virtual conference symposium. This real life experience, forced by the pandemic, can be the potential in changing the paradigm of medical education and meeting events. Ideally, a union between people and technology should be created. All the unlimited potential of the digital world should be integrated into the real world to form a 'hybrid' event. ${ }^{16}$ Outside of emergency situations, the overall uptake of telehealth has been slow. Adopting telemedicine or telehealth may be difficult because of costs, the challenges of high technology, lack of awareness, limited access to high-speed internet, reimbursement problems, and availability of technical support are the issues that may slow down the application of telehealth. ${ }^{17}$ In order to ensure that telemedicine is part of our routine practice we will need to ensure that all health professionals receive appropriate training. Funding which adequately covers the cost of providing telehealth needs to be addressed. As well, we need to support all professionals and decision makers with an effective communication and change management strategy and to establish systems to manage telehealth services on a routine basis. ${ }^{17}$ COVID-19 experience is not a first and will not be the last. While we may not be able to accurately predict the timing of natural disasters and infectious pandemics, we can be sure that they will present again in the future. It is important to develop telehealth strategy to deal with global and national emergency responses and for this to happen we need it built on the premise that telehealth becomes a mainstream component of our health system. ${ }^{14}$

\section{Research}

Ongoing clinical trials for existing drugs may provide further progress in limiting morbidity and mortality, yet vaccines are needed to prevent the spread of infections. Interestingly, following their epidemiological observational study, two urologists are leading one major clinical trial for a prophylaxis using BCG vaccine, namely BADAS trial (BCG As Defense Against SARS CoV-2)..$^{18,19}$ It is ironic that one of our oldest immunotherapies might help against the newest threat facing the globe. ${ }^{18}$ It is indeed prideworthy that urologists are exploring, and even leading, new fields in medicine beyond bladder cancer, particularly amid this pandemic crisis. On the other hand, CovidSurg ${ }^{20}$ is a research project that has been designed by an international collaborating group of surgeons and anesthetists. CovidSurg will be a platform of studies aiming to explore the impact of COVID-19 in surgical patients and services including;

I. The outcomes of surgery in patients diagnosed in COVID-19 
II. The safety of surgery for all types of cancer during the COVID-19 pandemic and the impact of the pandemic in cancer delay and treatment pathways

III. The influence of the COVID-19 pandemic on surgical services, particularly elective surgery cancellation and emergency surgery management
IV. The impact of COVID-19 on surgical workforce sickness, protection and task management

V. It is through data retrieved from such research work, we can adopt and adapt to a flexible attitude in management, to provide the best care possible to our patients and to be ready for any possible coming waves.

Table I Triaging urological patients awaiting surgery in the post COVID-19 era

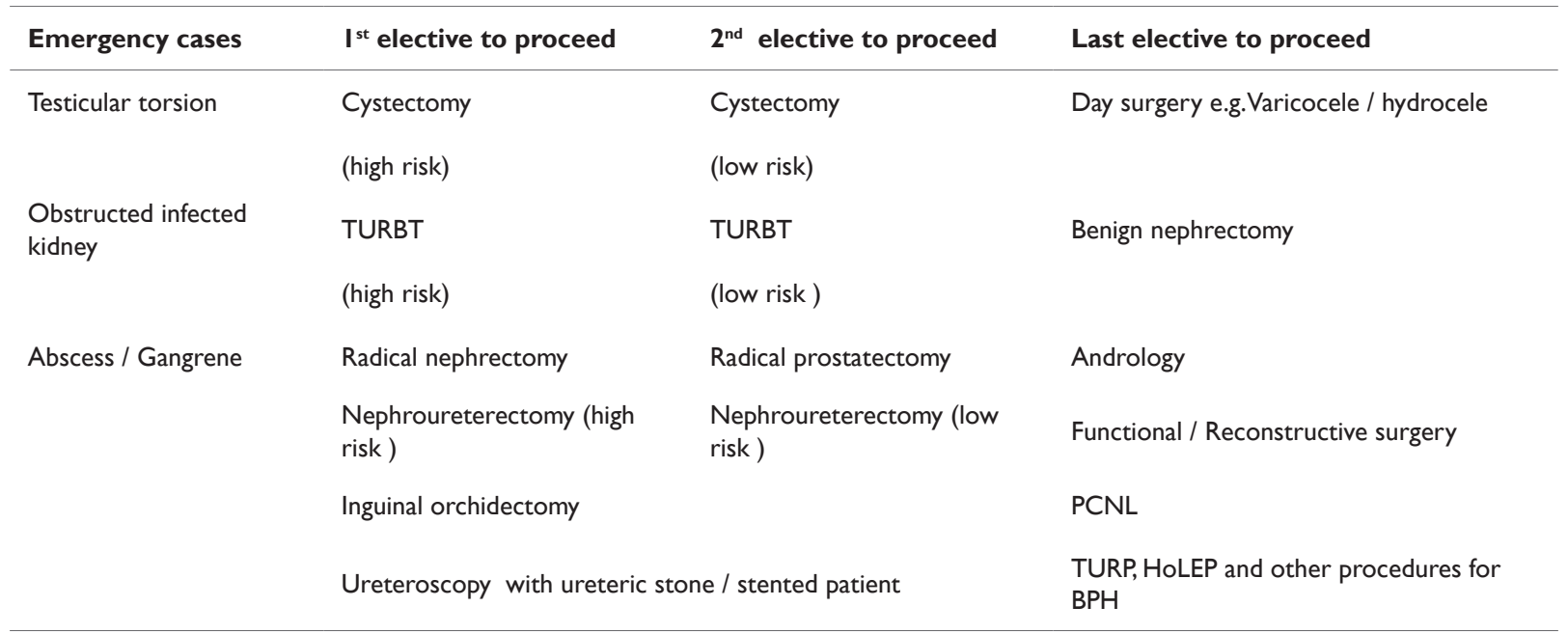

\section{Conclusion}

The lessons from COVID-19 are not stopping as we are still 'learning on the go' on daily basis from the bitter experiences around us. The after effect of this pandemic will last for a while after all is over. Urologists are an integral part of the health care system that is expected to recover from this crisis, resume activity and get prepared for any possible second waves. Getting this response right is in fact as important as what we are doing today.

\section{Acknowledgments}

None.

\section{Conflicts of interest}

The author has nothing to disclose.

\section{References}

1. Liu W, Chang S, Wang J, et al. Prolonged virus shedding even after seroconversion in a patient with COVID-19. J infect. 2020

2. Wu Y, Guo C, Tang L, et al. Prolonged presence of SARS-CoV-2 viral RNA in fecal samples. Lancet. 2020.

3. Zheng S, Fan J, Yu F, et al. Viral load dynamics and disease severity in patients infected with SARS-CoV-2 in Zhejiang province, China, January-March 2020: retrospective cohort study. BMJ. 2020;369:m1443.

4. Wang W, Xu Y, Gao R, et al. Detection of SARS-CoV-2 in different types of clinical specimens. JAMA. 2020323(18):1843-1844.

5. Ye G, Pan Z, Pan Y, et al. Clinical characteristics of severe acute respiratory syndrome coronavirus 2 reactivation. $J$ infect. 2020;80:14 17.
6. www.who.int/

7. Giulio M, Maggioni D, Montroni I, et al. Being a Doctor Will Never Be the Same After the COVID-19 Pandemic. Am J Med. 2020.

8. Clement LK. Chia. Being a Surgeon in the Pandemic Era. $J$ Am Coll Surg. 2020;230(6):1077-1079.

9. Coccolini F, Sartelli M, Kluger Y, et al. COVID-19 the showdown for mass casualty preparedness and management: the Cassandra Syndrome. World J Emerg Surg. 2020;15(1):26.

10. John Hick, Paul Biddinger. Novel Coronavirus and Old Lessons - Preparing the Health System for the Pandemic. $N$ Engl $J$ Med. 2020;382(20):e55.

11. Chan M, Yeo S, Chong Y, et al. Stepping Forward: Urologists' Efforts During the COVID-19 Outbreak in Singapore. Eur Urol. 202078(1):e38e39.

12. https://www.facs.org/covid-19/clinical-guidance/resuming-electivesurgery

13. www.BAUS.org.uk

14. Anthony S., Emma T., Centaine S. et al. Telehealth for global emergencies: Implications for coronavirus disease 2019 (COVID-19). Journal of telemedicine and telecare. 2020;26(5):309-313.

15. Luciani L, Mattevi D, Cai T, et al. Teleurology in the Time of Covid-19 Pandemic: Here to Stay? Urology. 2020;140:4-6.

16. Porpiglia F, Amparore D, Autorino R, et al. Traditional and Virtual Congress Meetings During the COVID-19 Pandemic and the PostCOVID-19 Era: Is it Time to Change the Paradigm? Eur Urol 2020;78(3):301-303.

17. Mahmoodpoor A, Akhbarzadeh M, Sanaie S, et al. Role of telehealth in outbreaks-Where the classical healthcare systems fail. Infect Control Hosp Epidemiol. 2020;41(8):992-994. 
18. Hegarty P, Sfakianos J, Giannarini G, et al. COVID-19 and Bacillus Calmette-Guérin: What is the Link? Eur Urol oncol. 2020;3(3):259-261.

19. https://www.urotoday.com/library-resources/bladder-cancer/120412beyond-bladder-cancer-bacillus-calmette-guerin-bcg-vaccinationrevisited-as-a-strategy-to-reduce-covid-19-hospitalization-in-theelderly.html?pk_campaign=Kamat_SocialLibResource_120412
20. https://globalsurg.org/covidsurg/ 